TRANSACTIONS OF THE

AMERICAN MATHEMATICAL SOCIETY

Volume 351 , Number 11, Pages 4411-4428

S 0002-9947(99)02308-9

Article electronically published on February 10, 1999

\title{
SOME INTERESTING NONSPHERICAL TEMPERED REPRESENTATIONS OF GRADED HECKE ALGEBRAS
}

\author{
C. KRILOFF
}

\begin{abstract}
Lusztig's presentation of the graded Hecke algebra in terms of generators and relations allows for the definition of algebras associated to noncrystallographic root systems. The representation theory of general graded Hecke algebras is investigated, the expected number of tempered representations for $\mathbb{H}\left(H_{3}\right)$ are accounted for, and it is shown that one of these representations has the unexpected property of being nonspherical despite being the only tempered representation appearing at its infinitesimal character. Additional nonspherical tempered representations of $\mathbb{H}\left(H_{4}\right)$ are also included.
\end{abstract}

\section{INTRODUCTION}

Using an algebraic definition of graded Hecke algebras, it is possible to define exceptional algebras associated to noncrystallographic root systems, whose representation theory differs somewhat from that of crystallographic graded Hecke algebras. We consider primarily the algebra associated to the root system of type $H_{3}$, which is closely related to the icosahedron or dodecahedron. In Section 7 we account for the tempered representations of this algebra that are expected by analogy with the crystallographic cases (indexed by the representations of the associated reflection group $W$ ). Theorem 7.3 illustrates how one of these representations differs in an important way from those that arise for the crystallographic algebras, since it is the only tempered representation that appears at its infinitesimal character and yet is nonspherical. We also mention four tempered representations of the algebra associated to the root system of type $H_{4}$ with this same surprising property.

Graded Hecke algebras originally arose as associated graded rings of affine Hecke algebras, defined as the compactly supported Iwahori-invariant functions on a reductive $p$-adic group. In this setting they are associated to crystallographic root systems and their representation theory has been well studied by Kazhdan and Lusztig [KL87, Lus88, Lus89, Lus95]. Utilizing instead the algebraic definition [Lus88], we introduce in Section 2 more general graded Hecke algebras, which include those associated to the noncrystallographic root systems of type $I_{2}(n), H_{3}$ and $\mathrm{H}_{4}$.

We then discuss in Sections 3-6 the adaptation of approaches used to study affine Hecke algebras [Kat82, Rog85, Che91] to the noncrystallographic case. These

Received by the editors December 1, 1997.

1991 Mathematics Subject Classification. Primary 16G99.

Supported by an NSF Graduate Research Fellowship and an Alfred P. Sloan Doctoral Dissertation Fellowship. 
include the reduction to classifying tempered representations (Langlands classification), the use of intertwining operators between principal series to develop a criterion for reducibility, and the parametrization of the constituents of certain reducible principal series. This parametrization is used to show that the principal series with the greatest reducibility have at least one tempered constituent.

In Section 7 we discuss the computer calculations which were used to show for the Hecke algebra $\mathbb{H}\left(H_{3}\right)$ that all such principal series have exactly one tempered constituent. This proves the main result accounting for 10 tempered representations of $\mathbb{H}\left(H_{3}\right)$. Similar calculations were used to verify for $\mathbb{H}\left(H_{4}\right)$ that four such principal series have exactly one tempered constituent. A more complete accounting for the dihedral graded Hecke algebras is given in [Kri].

An alternative means of studying graded Hecke algebras in this generality has been proposed by Heckman and Opdam [HO]. They consider a natural representation of $\mathbb{H}$ as an algebra of differential operators acting on a space of smooth functions. This allows them to obtain a classification of all spherical cuspidal representations of general graded Hecke algebras. Spherical refers to representations which contain the trivial representation on restriction to the reflection group $W$, and cuspidal refers to representations whose weights lie strictly within a cone defined by the fundamental co-weights (rather than possibly on the walls of the cone, as for tempered). These spherical cuspidal representations are related via an involution to the tempered representations presented below that contain the sign representation on restriction to $W$ and whose weights lie within this same cone.

Given this translation, for dihedral graded Hecke algebras the classification given in [Kri] and that given in [HO] are the same. The classification given by Heckman and Opdam in part involves finding distinguished points, which correspond precisely to the infinitesimal characters for the cuspidal representations, and then checking which of these will lead to spherical representations. For $\mathbb{H}\left(H_{3}\right)$ their list includes one nonspherical distinguished point, and the description given in Section 7 includes the nonspherical tempered representation which completes their classification. For $\mathbb{H}\left(H_{4}\right)$ their list includes four regular nonspherical distinguished points, which are used in the calculations that lead to the four tempered representations mentioned in Section 7. Thus Theorem 7.3 and Remark 7.4 provide five examples of representations which occur at infinitesimal characters for which the classification in [HO] yields no spherical cuspidal representations.

Another way of viewing the significance of these results is that they demonstrate an important difference between crystallographic and noncrystallographic graded Hecke algebras. The latter have the property that for each infinitesimal character at which there is a tempered representation, some tempered representation is spherical, which was originally formulated in terms of Whittaker models. Theorem 7.3 and Remark 7.4 show that this is not true for $\mathbb{H}\left(H_{3}\right)$ or $\mathbb{H}\left(H_{4}\right)$.

Most of the results included here were submitted as part of my doctoral thesis. I would like to express my deep appreciation to my thesis advisor, Allen Moy, as well as the other committee members. I am grateful to Eric Opdam, Chris Jantzen, and Mark Reeder for helpful conversations and John Stembridge for advice in using Maple. I would also like to thank the referee for suggesting the simple parametrization based on Rodier's Theorem that can be used both to show existence of tempered representations and to provide a criterion for determining whether representations are spherical. 


\section{Preliminaries}

We take as our starting point the definition of graded Hecke algebras in terms of generators and relations (see [Lus89] Proposition 4.4). For our purposes, we will require the following notion of a root system, which is just slightly different from that in [Lus89] and incorporates several of the associated objects which arise from the simpler definition in [Hum90].

Definition 2.1. A root system is a set of objects, $(\mathcal{X}, \mathcal{Y}, R, \check{R}, \Pi)$ such that

- $\mathcal{X}$ and $\mathcal{Y}$ are finite-dimensional real vector spaces with a non-degenerate pairing $\langle\rangle:, \mathcal{X} \times \mathcal{Y} \rightarrow \mathbb{R}$

- $R$ and $\check{R}$ are finite subsets of $\mathcal{X}$ and $\mathcal{Y}$ consisting of roots, $\alpha$, and co-roots, $\check{\alpha}$, with a bijection denoted ${ }^{`}$ between them such that $\langle\alpha, \check{\alpha}\rangle=2$, and

- $\Pi$ is a subset of $R$ consisting of the simple roots, $\left\{\alpha_{1}, \ldots, \alpha_{n}\right\}$, such that each $\alpha \in R$ has a unique expression as $\alpha=\sum_{i} c_{i} \alpha_{i}$ where all $c_{i} \geq 0$ or all $c_{i} \leq 0$. We say $\alpha$ is in $R^{+}$, the set of positive roots, or $R^{-}$, the set of negative roots.

These objects must satisfy the following two conditions.

1. For $\alpha \in R$, the only multiples of $\alpha$ in $R$ are $\pm \alpha$.

2. The reflections $s_{\alpha}: x \mapsto x-\langle x, \check{\alpha}\rangle \alpha$ (respectively $s_{\alpha}: y \mapsto y-\langle\alpha, y\rangle \check{\alpha}$ ) send $R$ to $R$ (respectively $\check{R}$ to $\check{R}$ ), for all $\alpha \in R$ (respectively $\check{\alpha} \in \check{R}$ ).

Such sets of data have been classified (see [Hum90]). We are primarily interested in the noncrystallographic root systems, which do not satisfy the assumption that the pairing of $\mathcal{X}$ and $\mathcal{Y}$ always takes values in $\mathbb{Z}$. The irreducible noncrystallographic root systems consist of $I_{2}(n)$ for $n=5$ and $n \geq 7, H_{3}$, and $H_{4}$, whose Coxeter diagrams are shown in Figure 1. We will use both $H_{3}$ and $H_{4}$ in Section 7. We will often assume that $\mathcal{X}$ is generated by $\Pi$. The fundamental co-weights are those $\omega_{j} \in \mathcal{Y}$ such that $\left\langle\alpha_{i}, \omega_{j}\right\rangle=\delta_{i j}$ for $\alpha_{i} \in \Pi$. An inner product $($,$) on \mathcal{X}$ can be used to identify the dual space $\mathcal{Y}$ with $\mathcal{X}$, so we write either $y(x)$ or $(x, y)$. Under this correspondence, $\check{\alpha}=\frac{2 \alpha}{(\alpha, \alpha)}$, and with the normalization $(\alpha, \alpha)=2$, we have $\check{\alpha}=\alpha$. Also associated with $R$ is the Coxeter group, $W$, generated by the simple reflections, $S=\left\{s_{i}=s_{\alpha_{i}} \mid i=1, \ldots, n\right\}$. For $H_{3}$, this group has presentation

$$
W=\left\langle s_{1}, s_{2}, s_{3} \mid s_{i}^{2}=s_{2}^{2}=s_{3}^{2}=\left(s_{1} s_{2}\right)^{5}=\left(s_{2} s_{3}\right)^{3}=\left(s_{1} s_{3}\right)^{2}=1\right\rangle,
$$

which has order 120 and is isomorphic to $A_{5} \times \mathbb{Z}_{2}$.

We can now define the general graded Hecke algebra associated to a root system $R$, denoted $\mathbb{H}(R)$ or just $\mathbb{H}$, in such a way that if $R$ is crystallographic, then $\mathbb{H}(R)$ can be obtained by grading an affine Hecke algebra.

Definition 2.2. As a complex vector space, the graded Hecke algebra $\mathbb{H}(R)=$ $\mathbb{C}[W] \otimes \mathbb{A} \otimes \mathbb{C}[r]$, where

- $\mathbb{C}[W]$ is the group algebra of the Coxeter group $W$ with generators $t_{w}$ for $w \in W$,

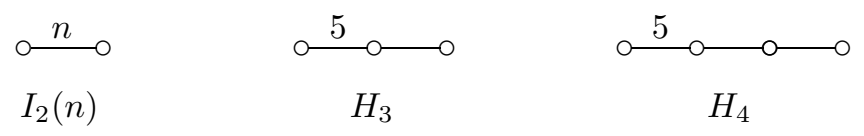

Figure 1. Coxeter diagrams 
- $\mathbb{A}$ is the symmetric algebra $S[\mathcal{Y}]$, or equivalently the ring of polynomial functions on $\mathcal{X}$ (this abelian algebra is canonically the polynomial ring $\mathbb{C}\left[y_{1}, \ldots, y_{n}\right]$ with generators the coordinate functions corresponding to the simple coroots), and

- $r$ is an indeterminant.

Using the contragredient action of $W$ on $\mathbb{A}$, the elements $t_{w} \in \mathbb{C}[W], p \in \mathbb{A}$, and $r$ are subject to the following relations:

- $r$ is central in $\mathbb{H}(R)$ (and is thus omitted from the relations),

- $\mathbb{C}[W] \hookrightarrow \mathbb{H}(R)$ via $t_{w} \mapsto t_{w} \otimes 1$ and $\mathbb{A} \hookrightarrow \mathbb{H}(R)$ via $p \mapsto 1 \otimes p$,

- $\left(t_{i} \otimes 1\right) \cdot(1 \otimes p)=t_{i} \otimes p$, and

$$
(1 \otimes p) \cdot\left(t_{i} \otimes 1\right)=\left(t_{i} \otimes 1\right) \cdot\left(1 \otimes s_{i}(p)\right)+2 r\left(1 \otimes \frac{\left(p-s_{i}(p)\right)}{\check{\alpha}_{i}}\right) \text { for } t_{i} \in W \text { and } p \in \mathbb{A} \text {. }
$$

Notice that this definition is more restrictive than that in [Lus89] in the sense that we have chosen parameters $c\left(\alpha_{i}\right)=c^{*}\left(\alpha_{i}\right)=1$ for all $i$. But it depends only on the root system, so it is possible to define the noncrystallographic algebras, including $\mathbb{H}\left(H_{3}\right)$. These algebras are of interest as they do not arise as the associated graded rings of affine Hecke algebras, which have already been investigated [KL87, Lus88, Lus89].

The final relation makes it easy to show that the center has a particularly nice form [Lus89]. The proof of this result does not depend on the crystallographic condition and thus extends immediately to noncrystallographic graded Hecke algebras.

Theorem 2.3 (Bernstein). The center of $\mathbb{H}$ is $\mathbb{A}^{W} \otimes \mathbb{C}[r]$, where $\mathbb{A}^{W}$ is the $W$ invariant polynomials of $\mathbb{A}$.

In practice, the final relation will be shortened to

$$
p \cdot t_{i}=t_{i} \cdot s_{i}(p)+2 r \frac{\left(p-s_{i}(p)\right)}{\check{\alpha}_{i}}=t_{i} \cdot s_{i}(p)+2 r p\left(\alpha_{i}\right) .
$$

In addition, we will specialize $r$ to a convenient nonzero value, chosen so that the formula is simple for $\mathbb{H}\left(A_{1}\right)$. Since the formula in this case is $y \cdot t=t \cdot(-y)+4 r, r$ will be taken to be $\frac{1}{4}$. As a vector space, $\mathbb{H}$ is $\mathbb{C}[W] \otimes \mathbb{A}$, and as an algebra $\mathbb{H}$ will have center $\mathbb{A}^{W}$.

We will denote the characters of $\mathbb{A}$ by $\mathcal{C}=\operatorname{Hom}(\mathbb{A}, \mathbb{C})$. As usual, for any finitedimensional $\mathbb{H}$-module, $V$, we have the decomposition $V=\bigoplus V_{\lambda}^{\text {gen }}$. Here $\lambda \in \mathcal{C}$ is a weight of $V$, meaning $V_{\lambda}=\{v \in V \mid p \cdot v=\lambda(p) v$ for all $p \in \mathbb{A}\} \neq 0$, and

$$
V_{\lambda}^{\text {gen }}=\left\{v \in V \mid(p-\lambda(p))^{k} \cdot v=0 \text { for all } p \in \mathbb{A}, \text { some } k \in \mathbb{Z}^{+}\right\} .
$$

By Theorem 2.3, a character of the center corresponds to a $W$-orbit, $W \chi$ for $\chi \in \mathcal{C}$, which is referred to as an infinitesimal character. On any irreducible $\mathbb{H}$-module, $V$, the center acts by a character, referred to as the infinitesimal character of $V$, and thus $\mathbb{H}$ being finite-dimensional over its center implies that $V$ is finite-dimensional. For this reason we will consider only finite-dimensional $\mathbb{H}$-modules.

In addition, a result in [BM93] indicates that in the crystallographic case it is sufficient to consider only graded Hecke algebras with real infinitesimal character. With this as motivation, we also consider only general graded Hecke algebras with real infinitesimal character. This accounts for not complexifying the spaces $\mathcal{X}$ and $\mathcal{Y}$ as is more typically done, and will in practice mean that $\chi \in \mathcal{C}$ will take values in $\mathbb{R}$ and will be identified with a vector in the real vector space $\mathcal{X}$. It also accounts for the fact that a finite number of tempered representations will be found rather than a finite number of infinite families of tempered representations. 


\section{LANGlands Classification}

We now give a brief exposition of a version of the Langlands classification, which reduces the representation theory of $\mathbb{H}$ to a classification of a particular type of representation known as tempered. The motivation for this approach comes from the $p$-adic setting, where the result can be stated for algebraic groups and then translated to affine Hecke algebras or crystallographic graded Hecke algebras, as in Theorem 6.6 in [BM93]. However, Evens [Eve96] has given a simplified algebraic proof for the graded Hecke algebras using highest weight methods. These do not depend on the crystallographic condition and thus extend to our setting. The essential nature of the result remains the same-to utilize tempered representations on parabolic subalgebras to describe all irreducible representations of graded Hecke algebras.

In describing these tempered representations and how they are induced from parabolic subalgebras, it will prove most useful to consider some decompositions. Let $\mathcal{X}=\mathfrak{a} \oplus \mathcal{X}_{s}$ and $\mathcal{Y}=\mathfrak{a}^{*} \oplus \mathcal{Y}_{s}$, where

$$
\begin{aligned}
\mathfrak{a} & =\{x \in \mathcal{X} \mid \check{\alpha}(x)=0 \text { for all } \alpha \in \Pi\}, \\
\mathfrak{a}^{*} & =\{y \in \mathcal{Y} \mid y(\alpha)=0 \text { for all } \alpha \in \Pi\}, \\
\mathcal{X}_{s} & =\left\{x \in \mathcal{X} \mid y(x)=0 \text { for all } y \in \mathfrak{a}^{*}\right\}, \\
\mathcal{Y}_{s} & =\{y \in \mathcal{Y} \mid y(x)=0 \text { for all } x \in \mathfrak{a}\} .
\end{aligned}
$$

If $R$ does not actually span $\mathcal{X}$ so that there is a nontrivial orthogonal part, then we can decompose $\mathbb{H}(R)$ as a pure tensor product $\mathbb{H}(R) \simeq \mathbb{H}_{s} \otimes S\left[\mathfrak{a}^{*}\right]$, where $\mathbb{H}_{s}$ is the graded Hecke algebra with root system $\left(\mathcal{X}_{s}, \mathcal{Y}_{s}, R, \check{R}, \Pi\right)$ such that $R$ does span $\mathcal{X}_{s}$. (The notation is motivated by the crystallographic case, where $\mathbb{H}_{s}$ arises from a Levi decomposition.) This decomposition becomes important in working with the parabolic subalgebra.

Definition 3.1. Fix any subset $P \subseteq\{1, \ldots, n\}$. Let $W_{P}$ be the parabolic subgroup generated by $\left\{s_{i} \mid i \in P\right\}$, let $R_{P}$ be generated by $\Pi_{P}=\left\{\alpha_{i} \mid i \in P\right\} \subseteq \Pi$, and let $\check{R}_{P}$ be generated by $\check{\Pi}_{P}=\left\{\check{\alpha}_{i} \mid \alpha_{i} \in \Pi_{P}\right\}$. The parabolic subalgebra, $\mathbb{H}_{P}(R)$, is the graded Hecke algebra with data $\left(\mathcal{X}, \mathcal{Y}, R_{P}, \check{R}_{P}, \Pi_{P}\right)$, or equivalently the subalgebra of $\mathbb{H}(R)$ with vector space structure $\mathbb{C}\left[W_{P}\right] \otimes \mathbb{A}$.

Now $R_{P}$ may not span $\mathcal{X}$ and we define $\mathfrak{a}_{P}$ and $\mathfrak{a}_{P}^{*}$ as before but with simple roots $\Pi_{P}$. For $p \in S\left[\mathfrak{a}_{P}^{*}\right]$ and $i \in P$ the relation is $p \cdot t_{i}=t_{i} \cdot s_{i}(p)+\frac{1}{2} p\left(\alpha_{i}\right)=t_{i} \cdot p$, so $S\left[\mathfrak{a}_{P}^{*}\right]$ lies in the center, $\mathbb{A}^{W_{P}}$, of $\mathbb{H}_{P}(R)$. We will denote by $\mathbb{H}_{P}$ the algebra $\mathbb{H}_{P}(R) \simeq \mathbb{H}_{s} \otimes S\left[\mathfrak{a}_{P}^{*}\right]$, where $\mathbb{H}_{s}=\mathbb{H}\left(R_{P}\right)$ has root system $\left(\mathcal{X}_{s}, \mathcal{Y}_{s}, R_{P}, \check{R}_{P}, \Pi_{P}\right)$.

In addition, an irreducible representation $U$ of $\mathbb{H}_{P}$ will split into a tensor product $U_{s} \otimes \mathbb{C}_{\nu}$ where $U_{s}$ is an irreducible representation of $\mathbb{H}_{s}$ and $\mathbb{C}_{\nu}$ is a one-dimensional representation of $S\left[\mathfrak{a}_{P}^{*}\right]$. In other words, $\nu: S\left[\mathfrak{a}_{P}^{*}\right] \rightarrow \mathbb{C}$ is a character, and by earlier remarks, we will take $\nu$ to map to $\mathbb{R}$. This type of splitting will lead to the parameter in the Langlands data and those characters will satisfy the additional condition used to define the following set:

$$
\mathfrak{a}_{P}^{+}=\left\{\nu \in \mathfrak{a}_{P} \mid(\nu, \check{\alpha})>0 \text { for all } \check{\alpha} \in \check{\Pi} \backslash \check{\Pi}_{P}\right\} .
$$

We now define the type of representation which will be of critical importance in classifying all irreducible representations of $\mathbb{H}$. 
Definition 3.2. An irreducible $\mathbb{H}_{P}$-module, $V$, is essentially tempered if $\left(\lambda, \omega_{i}\right) \leq$ 0 for all weights $\lambda$ of $V$ in $\mathcal{X}$ and all fundamental co-weights $\omega_{i}$ of $\mathbb{H}_{P}$. If in addition, $\left.\lambda\right|_{\mathfrak{a}_{P}^{*}}=0$ for all weights $\lambda$ of $V$, then $V$ is tempered.

The subalgebra $\mathbb{H}_{s}$ provides the most important information in determining whether a representation is tempered and the final condition on the restriction to $\mathfrak{a}_{P}^{*}$ should be viewed as a result of the decomposition of $\mathcal{Y}$. For example, when $P$ is $\{1, \ldots, n\}$ and $R$ spans $\mathcal{X}$, then $\mathfrak{a}_{P}=\mathfrak{a}_{P}^{*}=0$ so we can refer to any representation such that $\left(\lambda, \omega_{i}\right) \leq 0$ (respectively $\left(\lambda, \omega_{i}\right) \geq 0$ ) for all weights, $\lambda$, and all fundamental co-weights, $\omega_{i}$, as tempered (respectively antitempered).

It is now possible to state the main result.

Theorem 3.3. $\quad$ 1. Every irreducible $\mathbb{H}$-module, $V$, is a quotient of $\operatorname{Ind}_{\mathbb{H}_{P}}^{\mathbb{H}_{P}} U_{s} \otimes$ $\mathbb{C}_{\nu}$, where $U_{s}$ is a tempered $\mathbb{H}_{s}$-module and $\nu \in \mathfrak{a}_{P}^{+}$.

2. Every representation, $\operatorname{Ind}_{\mathbb{H}_{P}}^{\mathbb{H}} U_{s} \otimes \mathbb{C}_{\nu}$, where $U_{s}$ is a tempered $\mathbb{H}_{s}$-module and $\nu \in \mathfrak{a}_{P}^{+}$, has a unique irreducible quotient, denoted $J\left(P, U_{s}, \nu\right)$.

3. The data $\left(P, U_{s}, \nu\right)$ are unique: if $J\left(P, U_{s}, \nu\right) \simeq J\left(P^{\prime}, U_{s}^{\prime}, \nu^{\prime}\right)$, then $P=P^{\prime}$, $U_{s} \simeq U_{s}^{\prime}$, and $\nu=\nu^{\prime}$.

For the proof of this theorem, which involves methods of highest weight representations, see [Eve96].

Definition 3.4. Any representation $\operatorname{Ind}_{\mathbb{H}_{P}}^{\mathbb{H}} U_{s} \otimes \mathbb{C}_{\nu}$, as in Theorem 3.3 will be called a standard module and will be denoted $X\left(P, U_{s}, \nu\right)$.

\section{Principal SERIES}

While the standard modules are used for classifying irreducible representations, principal series will be used to account for tempered representations. These are also formed using parabolic induction, but from the minimal parabolic, $\mathbb{A}$, at a particular character $\chi \in \mathcal{C}$. They will contain copies of all of the tempered representations in a sense described below.

Definition 4.1. A principal series is a representation $I(\chi)=\operatorname{Ind}_{\mathbb{A}}^{\mathbb{H}} \chi=\mathbb{H} \otimes_{\mathbb{A}} \mathbb{C}_{\chi}$ for $\chi \in \mathcal{C}$.

In this definition, the $\mathbb{H}$-action can be explicitly described in terms of that for $\mathbb{C}[W]$ and $\mathbb{A}$. Take the set $\left\{\phi_{w}\right\}$ indexed by elements of $W$ as a basis for $I(\chi)$, and define $\phi_{w}=t_{w} \cdot \phi_{1}$, so that $t_{w^{\prime}} \cdot \phi_{w}=\left(t_{w^{\prime}} t_{w}\right) \cdot \phi_{1}=t_{w^{\prime} w} \cdot \phi_{1}=\phi_{w^{\prime} w}$ for $t_{w}, t_{w^{\prime}} \in$ $\mathbb{C}[W] \hookrightarrow \mathbb{H}$. In addition, let $p \cdot \phi_{1}=\chi(p) \phi_{1}$ for $p \in \mathbb{A} \hookrightarrow \mathbb{H}$, and extend the action to all of $\mathbb{H}$.

Notice that $I(\chi)$ is the universal $\mathbb{H}$-module with respect to having $\chi$-eigenvectors by Frobenius reciprocity. If $w$ is expressed as $s_{1} \cdots s_{n}$, then the relation defining $I(\chi)$ may be iterated to conclude that

$$
p \cdot t_{w}=t_{w} \cdot w^{-1}(p)+\sum_{v<w} t_{v} a_{v, w, p}
$$

where the $a_{v, w, p}$ are unique elements of $\mathbb{A}$. Since the action of $w \in W$ on $\chi$ is contragredient, for the module this becomes

$$
p . \phi_{w}=w \chi(p) \phi_{w}+\sum_{v<w} \chi\left(a_{v, w, p}\right) \phi_{v}
$$


The importance of this module is due in part to the fact that any irreducible finitedimensional $\mathbb{H}$-module is isomorphic to a submodule of some $I(\chi)$, which is easily shown using the existence of an $\mathbb{A}$-eigenvector and Frobenius reciprocity.

Proposition 4.2. Every irreducible finite-dimensional $\mathbb{H}-$ module, $V$, is a quotient module of $I(\chi)$ for some $\chi \in \mathcal{C}$.

Proof. Since $\mathbb{H}$ is finite-dimensional over its center $\mathbb{A}^{W}$, the action of $\mathbb{A}^{W}$ on any $V$ is by scalars, and there exists a nontrivial $\mathbb{A}$-eigenvector in $V$. By the adjoint property of induction and restriction, $\operatorname{Hom}(I(\chi), V) \simeq V_{\chi}$ and $V$, being irreducible, is a quotient of those $I(\chi)$ where $\chi$ is a weight of $V$. The existence of an $\mathbb{A}$ eigenvector then guarantees there is at least one such $I(\chi)$.

As we will see, the tempered representations arise as the constituents or irreducible composition factors of these principal series representations. It will be useful to observe that the weights of $I(\chi)$ are precisely the elements $\psi$ of $W \chi$ and for each of them, $\operatorname{dim}\left(I(\chi)_{\psi}^{\mathrm{gen}}\right)=\left|\operatorname{Stab}_{W}(\chi)\right|$, which is due to the generalized weight space decomposition for $I(\chi)$. Also of use in dealing with the intertwining operators below is the fact that $t_{w} \in \mathbb{C}[W]$ may be identified with $\phi_{w} \in I(\chi)$.

Principal series may also be analyzed more precisely for the appearance of particular irreducible representations. Utilizing intertwining operators, Kato [Kat82] and Rogawski [Rog85] have both given characterizations of irreducible principal series representations for affine Hecke algebras. Cherednik [Che91] has extended the discussion of these results to the case of graded Hecke algebras associated to crystallographic root systems, but does not use the crystallographic condition. Here we present statements of the primary results in the setting of graded Hecke algebras associated to noncrystallographic root systems. Many of the proofs will be omitted because they are largely the same as those in [Rog85], but mention will be made of an important modification, due to Cherednik.

We will use as an intertwining operator right multiplication by the element $A_{s_{i}}(\chi)=-1+b_{\chi, s_{i}} t_{i} \in \mathbb{C}[W]$ where $s_{i} \in S$ and $b_{\chi, s_{i}}=\chi\left(y_{i}-s_{i}\left(y_{i}\right)\right)=2 \chi\left(\check{\alpha}_{i}\right)$. This is chosen to guarantee that $\tilde{A}_{s_{i}}(\chi)$, right multiplication by $A_{s}(\chi)$, is an intertwining operator from $I\left(s_{i} \chi\right)$ to $I(\chi)$. We can then define the element $A_{w}(\chi)$ in terms of a reduced decomposition $s_{1} \cdots s_{m}$ of $w$ as the product $A_{s_{1}}\left(s_{2} \cdots s_{m} \chi\right)$. $A_{s_{2}}\left(s_{3} \cdots s_{m} \chi\right) \cdots A_{s_{m}}(\chi)$ and $\tilde{A}_{w}(\chi)$ as $\tilde{A}_{s_{m}}(\chi) \circ \cdots \circ \tilde{A}_{s_{1}}\left(s_{2} \cdots s_{m} \chi\right)$ so that $A_{w}(\chi)=\tilde{A}_{w}(\chi) \phi_{1}$. Following through this composition yields that $\tilde{A}_{w}(\chi)$ is an intertwining operator from $I(w \chi)$ to $I(\chi)$ and that we can view $A_{w}(\chi)$ as an element of $I(\chi)_{w \chi}$. This is independent of the choice of reduced decomposition for $w$ when $\operatorname{dim} I(\chi)_{w \chi}=1$, for example if $\operatorname{Stab}_{W} \chi=1$.

It is straightforward to provide a criterion for which of these intertwining operators are invertible.

Proposition 4.3. For any character, $\chi$, and reduced decomposition, $s_{1} \cdots s_{m}$, of $w, \tilde{A}_{w}(\chi)$ is invertible if and only if $s_{j+1} \cdots s_{m} \chi\left(\check{\alpha}_{j}\right) \neq \pm \frac{1}{2}$ for all $j=1, \ldots, m-1$.

Proof. First consider $\tilde{A}_{s}(\chi)$. Choose a basis for $\mathbb{C}[W]$, and using the action of $t_{s}$, break it into two-dimensional subspaces spanned by $\left\{\phi_{w}, \phi_{s w}\right\}$. The action of $b_{\chi, s} t_{s}$ on each subspace is given by $\left(\begin{array}{cc}0 & b_{\chi, s} \\ b_{\chi, s} & 0\end{array}\right)$. Changing basis so that the twodimensional subspaces are spanned by $\left\{\phi_{w}+\phi_{s w}, \phi_{w}-\phi_{s w}\right\}$, the action on each 
subspace becomes

$$
\left(\begin{array}{cc}
b_{\chi, s} & 0 \\
0 & -b_{\chi, s}
\end{array}\right)
$$

Hence

$$
\tilde{A}_{s}(\chi)=\left(\begin{array}{ccccc}
-1+b_{\chi, s} & 0 & \cdots & & 0 \\
0 & -1-b_{\chi, s} & & & \\
\vdots & & \ddots & & \\
0 & & & -1+b_{\chi, s} & 0 \\
0 & & & 0 & -1-b_{\chi, s}
\end{array}\right)
$$

which is clearly invertible precisely when $b_{\chi, s}=2 \chi\left(\check{\alpha}_{s}\right) \neq \pm 1$.

Now $\tilde{A}_{w}(\chi)$ will be invertible if and only if each $\tilde{A}_{s_{j}}\left(s_{j+1} \cdots s_{m} \chi\right), j=1, \ldots, m-$ 1 is invertible, yielding the desired condition.

This is then used in showing the invariance of the principal series under the action of $W$ on the inducing character.

Proposition 4.4. For all $\chi \in \mathcal{C}$ and $w \in W$, the composition factors for $I(\chi)$ and $I(w \chi)$ are the same.

The proof involves reduction to the case when $w$ is a simple reflection followed by a straightforward analysis of the invertibility of the intertwining operator $\tilde{A}_{w}(\chi)$. A consequence is that it is only necessary to analyze principal series induced from characters in a single chamber.

\section{REDUCIBILITY OF PRINCIPAL SERIES}

We continue toward a description of which principal series are irreducible by proving that certain weight spaces are one-dimensional. This frequently involves reducing to a special character.

Definition 5.1. A character $\psi \in \mathcal{C}$ is special if $\operatorname{Stab}_{W}(\psi)$ is generated by simple reflections.

Note that since we are assuming that all infinitesimal characters are real, we have that for every $\chi$, some element, $\psi$, of $W \chi$ is special.

The next two lemmas will be used in the proof of the main result, Theorem 5.4, which has been somewhat altered from that in [Rog85]. The first will be used for evaluation at the zero character, $\chi_{0}$, after reducing to the case of a special character by means of the second lemma. The first lemma is essentially due to Cherednik, with some slight modifications, and will require some results related to the structure of $\mathbb{A}$, which can be found in Chapter III of [Hel84].

Recall that we are viewing $\mathbb{A}$ as the symmetric algebra, $S[\mathcal{Y}]$, or polynomial algebra, $\mathbb{C}[\mathcal{X}]$. We will use the nondegenerate symmetric bilinear form on $\mathbb{A}$ given by $\langle p, q\rangle=\partial_{p}(q)(0)$ where $\partial_{p}$ is the constant coefficient differential operator determined by the polynomial $p$. This form has the property that $\left\langle y_{i} p, q\right\rangle=\left\langle p, \partial_{i} q\right\rangle$ for $\partial_{i}=$ $\partial_{y_{i}}$. Let $\mathfrak{m}$ be the ideal of $\mathbb{A}$ generated by the homogeneous elements of $\mathbb{A}^{W}$ of positive degree. Then $\mathbb{A}$ can be decomposed as $\mathfrak{m}+H$, where $H$ is the orthogonal 
complement of $\mathfrak{m}$ with respect to the above form, or as $H \otimes \mathbb{A}^{W}$ (see Theorem 1.1 in [Hel84]). This subalgebra, $H$, of harmonic polynomials is in fact spanned by the element $d=\prod_{\alpha \in R^{+}} y_{\alpha}$ and its derivatives $\partial_{p}(d)$, where $p \in \mathbb{A}$ (see Theorem 3.6 in $[\mathrm{Hel} 84])$.

Lemma 5.2. The principal series $I\left(\chi_{0}\right)$ is irreducible and $I\left(\chi_{0}\right)_{0}$ is one-dimensional.

Proof. This will be proven by constructing a concrete "model" for the $\mathbb{H}$-module $I\left(\chi_{0}\right)$. Let $V^{0}=\operatorname{Ind} \underset{\mathbb{C}[W]}{\mathbb{H}}$ triv $\simeq \mathbb{A}$ and $\mathfrak{m}$ be as described above. Then we claim that the quotient module $V_{\mathfrak{m}}^{0}=V^{0} / \mathfrak{m} V^{0}$ is isomorphic to $I\left(\chi_{0}\right)$.

Observe that for $d=\prod_{\alpha \in R^{+}} y_{\alpha} \in V^{0}$, the element $\bar{d} \in V_{\mathfrak{m}}^{0}$ is sent to 0 under the action of all $y_{i}$. This is clear using the decomposition $\mathbb{A}=\mathfrak{m} \oplus H$, since $y_{i} d$ is a homogeneous polynomial of degree greater than $\left|R^{+}\right|$and thus lies in $\mathfrak{m}$. Universality now provides a nonzero homomorphism, $\sigma$, from $I\left(\chi_{0}\right)$ to $V_{\mathfrak{m}}^{0}$ sending $\phi_{1}$ to $\bar{d}$.

We will prove $\sigma$ is surjective by showing $V_{\mathfrak{m}}^{0}=\mathbb{H} \bar{d}$ using a result of Demazure and Bernstein, Bernstein, and Gelfand described in [Hil82]. This involves an inductive step for which it will be useful to define operators $\Delta_{i}$ on $\mathbb{A}$ by rearranging the final relation in the definition of $\mathbb{H}$ as $t_{i} \cdot p=s_{i}(p) \cdot t_{i}+\frac{1}{2} \Delta_{i}(p)$, so that $\Delta_{i}(p)=$ $\left(p-s_{i}(p)\right) / \check{\alpha}_{i}$.

To show $V_{\mathfrak{m}}^{0}=\mathbb{H} \bar{d}$ it is sufficient to find an element with constant term, or in other words, of the form $f=1+f_{0} \in \mathbb{H} d$ where $f_{0}(0)=0$. This is because we can then act by the elements $h \in H$ such that $\bar{h}$ are the generators for $V_{\mathfrak{m}}^{0}$ and see that the various $\overline{h f} \in \mathbb{H} \bar{d}$ generate all of $V_{\mathfrak{m}}^{0}$.

Second, we find such an element by using the composition, $\Delta_{0}$, of the operators $\Delta_{i}$ according to a reduced expression for the longest element $w_{0} \in W$. Note that if $p$ is a homogeneous polynomial of degree $k$ in the induced module $V^{0}$, then $t_{i} \cdot p=s_{i}(p)+\frac{1}{2} \Delta_{i}(p)$ with $s_{i}(p)$ a homogeneous polynomial of degree $k$ and $\Delta_{i}(p)$ a homogeneous polynomial of degree $k-1$. By Proposition 1.6 in [Hil82], $\Delta_{0}$ is well-defined and $\Delta_{0}(d)$ is the scalar $|W|$. Thus, the element $t_{w_{0}} . d \in \mathbb{H} d$ has a constant term, since it is of the form $\Delta_{0}(d)+d_{0}$, with $d_{0}(0)=0$, and $\sigma$ is surjective. Furthermore, $\operatorname{dim} I\left(\chi_{0}\right)=\operatorname{dim} V_{\mathfrak{m}}^{0}=|W|$, so $\sigma$ is an isomorphism.

To see that $V_{\mathfrak{m}}^{0}$ is irreducible, it is sufficient to show that $\bar{d}$ is the unique element, up to scalar multiple, such that $y_{i} \bar{d}=0$ for all $i$. This is sufficient because any submodule of $V_{\mathrm{m}}^{0}$ must contain an element with $\mathbb{A}$-eigenvalue 0 , and thus will contain $\bar{d}$ and be all of $V_{\mathfrak{m}}^{0}$. Now suppose for some $u \in H$ that $y_{i} u \in \mathfrak{m} V^{0}$ for all $i$. Then by the characterization of $H$ as $\mathfrak{m}^{\perp}$, this means $\left\langle y_{i} u, q\right\rangle=0$ for all $i$ and all $q \in H$. In other words, $\left\langle u, \partial_{i} q\right\rangle=0$ for all $i$ and all $q \in H$. But using that $H$ is spanned by $d$ and $\partial_{p}(d)$ and that $\mathfrak{m}=H^{\perp}$ (Lemma 3.9 in [Hel84]), we see that this implies $u \in \mathbb{C} d$ and $\bar{u} \in \mathbb{C} \bar{d}$. This also shows $I\left(\chi_{0}\right)_{0}$ is one-dimensional, generated by $\phi_{1}$.

Lemma 5.3. If $\psi$ is special, then $I(\psi)_{\psi}^{g e n}$ is an irreducible $\mathbb{H}^{\prime}$-module, where $\mathbb{H}^{\prime}$ is the parabolic subalgebra of $\mathbb{H}$ generated by $\left\{t_{w} \mid w \in \operatorname{Stab}_{W}(\psi)\right\}$ and $\mathbb{A}$.

Proof. We claim that $I(\psi)_{\psi}^{\text {gen }}=\operatorname{Span}\left\{h . \phi_{1} \mid h \in \mathbb{H}^{\prime}\right\}$, which allows us to view it as an $\mathbb{H}^{\prime}$-submodule. First we show that $\operatorname{Span}\left\{h . \phi_{1}\right\} \subset I(\psi)_{\psi}^{\text {gen }}$. For $h \in \mathbb{A}$, it is clear that $(p-\psi(p)) h . \phi_{1}=h(p-\psi(p)) . \phi_{1}=0$ and $h . \phi_{1} \in I(\psi)_{\psi}^{\text {gen }}$. Certainly $\phi_{1} \in I(\psi)_{\psi}^{\text {gen }}$ and for $h=t_{w} \in \mathbb{C}\left[\operatorname{Stab}_{W}(\psi)\right]$, we see $h . \phi_{1} \in I(\psi)_{\psi}^{\text {gen }}$ by induction 
since

$$
\begin{aligned}
(p- & \psi(p))^{k} \cdot t_{w} \cdot \phi_{1}=(p-\psi(p))^{k-1} \cdot\left[t_{w} \cdot w^{-1}(p)+\sum_{v<w} t_{v} a_{v, w, p}-\psi(p) t_{w}\right] \cdot \phi_{1} \\
& =(p-\psi(p))^{k-1} \cdot\left[w \psi(p) t_{w} \cdot \phi_{1}-\psi(p) t_{w} \cdot \phi_{1}+\sum_{v<w} \psi\left(a_{v, w, p}\right) t_{v} \cdot \phi_{1}\right] \\
& =(p-\psi(p))^{k-1} \cdot \sum_{v<w} \psi\left(a_{v, w, p}\right) t_{v} \cdot \phi_{1} .
\end{aligned}
$$

The reverse containment follows by counting, since $\operatorname{dim} I(\psi)_{\psi}^{\mathrm{gen}}=\left|\operatorname{Stab}_{W}(\psi)\right|$.

In addition, $I(\psi)_{\psi}^{\text {gen }}$ is irreducible as an $\mathbb{H}^{\prime}$-module. Let $S^{\prime}=\operatorname{Stab}_{W}(\psi) \cap S$ be a generating set for $\operatorname{Stab}_{W}(\psi)$ with index set $P^{\prime}$. As in Section 3, this splits $\mathcal{X}$ into $\mathfrak{a}_{P^{\prime}} \oplus \mathcal{X}_{s}$ and $\mathcal{Y}$ into $\mathfrak{a}_{P^{\prime}}^{*} \oplus \mathcal{Y}_{s}$ and $\mathbb{A}$ into $\mathbb{A}_{S^{\prime}} \otimes \mathbb{A}_{S^{\prime}}^{\perp}=S\left[\mathfrak{a}_{P^{\prime}}^{*}\right] \otimes S\left[\mathcal{Y}_{s}\right]$, so that $\mathbb{H}^{\prime}=\mathbb{H}_{P^{\prime}}\left(R^{\prime}\right) \otimes S\left[\mathfrak{a}_{P^{\prime}}^{*}\right]$, where $R^{\prime}$ is the root system generated by $\left\{\alpha_{i} \mid i \in P^{\prime}\right\}$. This allows $I(\psi)_{\psi}^{\text {gen }}$ to be viewed as $\left.\operatorname{Ind}_{A_{S^{\prime}}}^{\mathbb{H}_{P^{\prime}}\left(R^{\prime}\right)} \chi_{0} \otimes \psi\right|_{\mathbb{A}_{S^{\prime}}}$. But by Lemma 5.2, $I\left(\chi_{0}\right)$ is irreducible. In addition, $I\left(\chi_{0}\right)_{0}$ is one-dimensional, as is $\left.\psi\right|_{\mathbb{A}_{S^{\prime}}}$. Thus $I(\psi)_{\psi}^{\text {gen }}$ is irreducible.

Theorem 5.4. If $\psi \in W \chi$ is special, then $I(\chi)_{\psi}$ is one-dimensional.

Proof. It is sufficient to prove this when $\psi=\chi$ by the following reasoning. Suppose $\operatorname{dim} I(\psi)_{\psi}=1$. Since $\psi \in W \chi, I(\chi)$ contains a vector with weight $\psi$. Thus, by universality there exists a nonzero map from $I(\psi)$ to $I(\chi)$, which can be restricted to a map from $I(\psi)_{\psi}^{\text {gen }}$ to $I(\chi)_{\psi}^{\text {gen }}$. This map is injective, since $\psi$ being special implies, by Lemma 5.3 , that $I(\psi)_{\psi}^{\text {gen }}$ is irreducible. In addition, these modules have the same dimension, $\left|\operatorname{Stab}_{W}(\chi)\right|$, so they are isomorphic and $I(\chi)_{\psi} \simeq I(\psi)_{\psi}$ has dimension one.

Now suppose that $\chi$ is special and $\operatorname{dim} I(\chi)_{\chi}>1$. We shall exhibit an element, $\phi^{P} \notin \mathbb{C} \phi_{1}$, of a parabolic subalgebra with eigenvalue 0 , which will contradict Lemma 5.2.

First, the hypothesis that $\chi$ is special implies that $\operatorname{Stab}_{W}(\chi)$ is a parabolic subgroup of $W$, whose generators correspond to roots in a possibly reducible subroot system of that for $\mathbb{H}$. Thus we have not only the decomposition from the proof of Lemma 5.3, $I(\chi)_{\chi}^{\text {gen }}=\bigoplus \mathbb{C} t_{w}$ with $w \in \operatorname{Stab}_{W}(\chi)$, but also the decomposition $I(\chi)_{\chi}^{\text {gen }}=\bigotimes_{P} \mathbb{C}\left[W_{P}\right]$, where $\operatorname{Stab}_{W}(\chi)=\prod_{P} W_{P}$ and the various $P$ index the connected components of the subroot system.

Second, if $\operatorname{dim} I(\chi)_{\chi}>1$, then there exists $\phi \notin \mathbb{C} \phi_{1}$ with $\phi \in I(\chi)_{\chi}$. Choose $P$ so that, using the second decomposition, $\phi=\sum_{j} \phi_{j} \hat{\phi}_{j}$, such that $\phi_{j} \in \mathbb{C}\left[W_{P}\right]$, $\hat{\phi}_{j} \in \prod_{P^{\prime} \neq P} \mathbb{C}\left[W_{P^{\prime}}\right]$ and for some fixed $j, \phi_{j} \notin \mathbb{C} \phi_{1}$. Since $\phi \in I(\chi)_{\chi}$, for $k \in P$, we have $y_{k} . \phi=\sum_{j} y_{k} \cdot \phi_{j} \hat{\phi}_{j}=\sum_{j} \chi\left(y_{k}\right) \phi_{j} \hat{\phi}_{j}=0$. In particular, since the generators for the abelian subalgebra correspond to co-roots, $y_{k} \cdot \phi_{j}=0$ for $\phi_{j} \in \mathbb{H}_{P}$ and $k \in P$. The existence of this element $\phi^{P}=\phi_{j}$ provides the necessary contradiction to the assumption that $\operatorname{dim} I(\chi)_{\chi}>1$.

The first corollary of this theorem gives us the main result on which of the principal series reduce. 
Corollary 5.5. The principal series $I(\chi)$ is irreducible if and only if $w \chi\left(\check{\alpha}_{i}\right) \neq \pm \frac{1}{2}$ for all $w \in W$ and $i=1, \ldots, n$.

Proof. See the proof of Corollary 3.2 in [Rog85].

Thus "most" principal series are irreducible and those that are reducible are induced from characters which lie along the hyperplanes of reducibility described in Corollary 5.5. Furthermore, only one of the irreducible principal series is tempered.

Proposition 5.6. The only irreducible principal series which is tempered is $I\left(\chi_{0}\right)$.

Proof. Clearly $I\left(\chi_{0}\right)$ is tempered and we have shown it is irreducible. Suppose $\chi \neq 0$ and $I(\chi)$ is irreducible. Then the weights of $I(\chi)$ are $\{w \chi \mid w \in W\}$ and for some $w \in W, w \chi$ will lie in the antitempered positive cone $A$. Since $I(\chi)$ is irreducible, it is not tempered.

Hence it is sufficient to analyze only reducible principal series in order to find further tempered representations.

\section{RODIER's THEOREM}

We now state a second corollary of Theorem 5.4 which will be used to prove a version of a theorem due to Rodier.

Corollary 6.1. If $\chi$ is special, $I(\chi)$ has a unique irreducible quotient which is the unique constituent of $I(\chi)$ with $\chi$ as a weight.

Proof. It is easy to show that any submodule $U$ is proper if and only if $U_{\chi}^{\text {gen }}=$ 0 , using Theorem 5.4 and the parabolic subalgebra of $\mathbb{H}$ generated by $\mathbb{A}$ and $\left\{t_{w} \mid w \in \operatorname{Stab}_{W}(\chi)\right\}$. Since the sum of any two proper submodules is proper, there is a unique maximal proper submodule of $I(\chi)$, and hence a unique irreducible quotient.

We will use the following definition for the Cayley graph of a group. As an example, the Cayley graph for the Coxeter group of type $H_{3}$ appears in Section 7 , Figure 2.

Definition 6.2. The Cayley graph, $\Gamma$, of a group $W$ generated by $S$ and having elements $\left\{w_{k}\right\}$, has vertices corresponding to the elements $w_{k}$ and an edge with label $i$ between $w_{k}$ and $w_{l}$ if $w_{k}=s_{i} w_{l}$ for some $s_{i} \in S$.

For the following theorem we will also need the definitions of regularity and formal character.

Definition 6.3. A character $\chi \in \mathcal{C}$ and an infinitesimal character with representative $\chi$ are said to be regular if $\operatorname{Stab}_{W}(\chi)=1$.

Definition 6.4. For any finite-dimensional $\mathbb{H}$-module $V$, the formal character is viewed as being in the integral group ring of characters of $\mathbb{A}$, and is defined as

$$
\operatorname{ch}(V)=\sum_{\chi \in \mathcal{C}}\left(\operatorname{dim}\left(V_{\chi}^{\text {gen }}\right)\right) \chi
$$

Theorem 6.5 (Rodier). Let the graph $\Gamma(\chi)$ be obtained from the Cayley graph of $W$ by removing edges

$$
\stackrel{i}{\stackrel{i}{\longrightarrow}} \stackrel{s_{i} w}{\circ}
$$


such that $w \chi\left(\check{\alpha}_{i}\right)=\frac{1}{2}$. If $\chi$ is regular, then there is a bijection between connected components of $\Gamma(\chi)$ and constituents of $I(\chi)$. Furthermore,

$$
\operatorname{ch}\left(V_{k}\right)=\sum_{w \in \hat{\Gamma}_{k}(\chi)} w \chi
$$

where $V_{k}$ is a constituent of $I(\chi)$ and $\hat{\Gamma}_{k}(\chi)$ is the set of vertices of the corresponding connected component of $\Gamma(\chi)$.

Proof. The proof of this theorem proceeds as in Proposition 3.5 in [Rog85] with a few minor alterations. The initial step is a reduction to one constituent corresponding to the unique irreducible quotient of $I(\chi)$ with weight $\chi$, which requires Corollary 6.1, (this applies since $\chi$ is regular and thus special). By Proposition 4.4, this constituent may be taken to be $V_{1}$. Proposition 4.3 then provides the connection between invertibility of $A_{w}(\chi)$ and $w$ being in $\hat{\Gamma}_{1}(\chi)$.

Remark 6.6. It is useful to also mention the version of this parametrization originally developed by Rodier for $p$-adic group representations [Rod81]. There the constituents were parametrized by the connected components of $\mathcal{Y} \backslash \bigcup_{j=1}^{m} \operatorname{Ker} \check{\beta}_{j}$ where the union is over all co-roots $\check{\beta}_{j}$ (not necessarily simple) with $\chi\left(\check{\beta}_{j}\right)=\frac{1}{2}$ (i.e. the various $w^{-1}\left(\alpha_{i}\right)$ that satisfy the condition given in the theorem). These components consist of translates, $w C^{+}$, of the (open) fundamental Weyl chamber together with interior walls. The translates contained in one component all lie on the same sides of all $\operatorname{Ker} \check{\beta}_{j}$, or equivalently, all $w^{-1}\left(\beta_{j}\right)$ are of the same sign for each fixed $\beta_{j}$. Similarly, the connected components of the altered Cayley graph defined above consist of vertices $w \in W$ such that all $w\left(\beta_{j}\right)$ have the same sign for each fixed $\beta_{j}$ (the Cayley graph and the translates are related by $w \mapsto w^{-1}$ ). Thus it is clear that the constituents can equivalently be indexed by sequences $\left(e_{1}, \ldots, e_{m}\right)$ with $e_{j}=\operatorname{sign}\left(w \beta_{j}\right)$ for any $w$ such that $w \chi$ is a weight of the constituent.

Now suppose that $m=n$ and express $\chi=\sum_{j=1}^{n} c_{j} \check{\beta}_{j}$ as a linear combination of the co-roots $\check{\beta}_{j}$ where $c_{j} \in \mathbb{R}$. Consider the constituent indexed by the sequence of signs opposite those of the coefficients $c_{j}$. Since $\left(w \chi, \omega_{i}\right)=\sum_{j=1}^{n} c_{j}\left(w \check{\beta}_{j}, \omega_{i}\right) \leq 0$ for all fundamental co-weights $\omega_{i}$, this constituent is tempered. Recall from the introduction that we refer to a representation as spherical if it contains the sign representation on restriction to $W$. A constituent is spherical if and only if its index sequence is $e_{j}=+$ for all $j$. This makes it clear that at least one constituent in each fully reducible principal series is tempered, and allows us to detect any non-spherical tempered constituents.

\section{TEMPERED Representations of $\mathbb{H}\left(H_{3}\right)$ And $\mathbb{H}\left(H_{4}\right)$}

Rodier's Theorem, the remark following it, and the results in Section 5 can be used to account for the expected number of tempered representations of $\mathbb{H}\left(H_{3}\right)$ by investigating constituents of principal series with certain infinitesimal characters. Finding dimensions of these constituents and verifying that there are no additional tempered constituents at the infinitesimal characters requires finding all weights and determining the parametrization in Rodier's Theorem. This is computationally involved for $H_{3}$, so the calculations were carried out using the software package 
Maple. Before indicating how this was done, we explicitly list some normalizations. We use the following simple roots and corresponding fundamental co-weights for the root system $\mathrm{H}_{3}$, whose roots lie at the midpoints of the edges of an icosahedron. These are written with respect to the standard basis of $\mathbb{R}^{3}$, with $\beta=\cos \frac{\pi}{5}$ and $\gamma=\cos \frac{2 \pi}{5}$.

$$
\begin{array}{rlrl}
\alpha_{1} & =\sqrt{2}\left(\beta,-\frac{1}{2}, \gamma\right), & \omega_{1} & =\sqrt{2}(\beta, \beta, \beta), \\
\alpha_{2}=\sqrt{2}\left(-\beta, \frac{1}{2}, \gamma\right), & \omega_{2} & =\sqrt{2}\left(\frac{1}{2}, \beta+\frac{1}{2}, \beta\right), \\
\alpha_{3} & =\sqrt{2}\left(\frac{1}{2}, \gamma,-\beta\right), & \omega_{3} & =\sqrt{2}\left(\frac{1}{2}, \beta, 0\right) .
\end{array}
$$

The reflection group for $H_{3}$ has presentation

$$
W=\left\langle s_{1}, s_{2}, s_{3} \mid s_{i}^{2}=s_{2}^{2}=s_{3}^{2}=\left(s_{1} s_{2}\right)^{5}=\left(s_{2} s_{3}\right)^{3}=\left(s_{1} s_{3}\right)^{2}=1\right\rangle,
$$

with order 120 and 10 conjugacy classes. The Cayley graph (shown in Figure 2) corresponding to $W \simeq$ Alt $_{5} \times \mathbb{Z}_{2}$ can be constructed using the procedures cayley_graph and plot_cayley written by Stembridge [Ste92]. A procedure called traverse returns a list of reduced expressions for the elements of $W$ which can be used to generate the reflection representation of the group.

In accounting for tempered representations of $\mathbb{H}\left(H_{3}\right)$, we first notice that $\mathbb{H}\left(H_{3}\right)$ has seven proper parabolic subalgebras. We may induce tempered representations from each of these subalgebras with parameter $\nu=0$, which yields the following list of six representations of $\mathbb{H}\left(H_{3}\right)$. The Steinberg representation, St, is the sign representation on $\mathbb{C}[W]$ and has unique extension to $\mathbb{A}$. The representation $\tau_{1}$ is the only tempered representation used that is not the Steinberg representation or a representation that is itself induced. It can be found by applying Rodier's Theorem

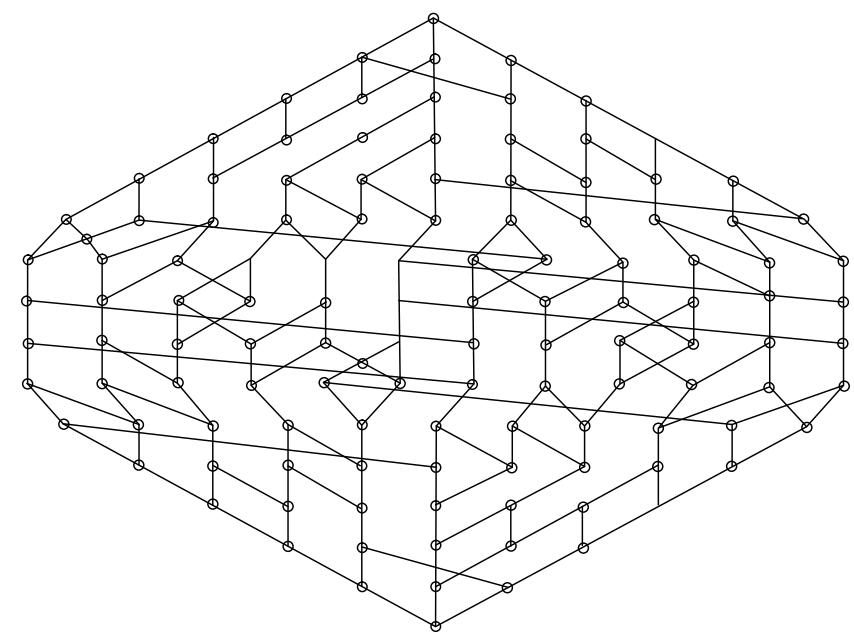

Figure 2. Cayley graph $\Gamma$ of $\mathrm{Alt}_{5} \times \mathbb{Z}_{2}$ 
at the one intermediate regular character of $\mathbb{H}\left(I_{2}(5)\right)$.

$$
\begin{array}{ll}
\operatorname{Ind}_{\mathbb{A}}^{\mathbb{H}} \chi_{0}, & \operatorname{Ind}_{\mathbb{H}_{1}}^{\mathbb{H}} S t \otimes \chi_{0} \simeq \operatorname{Ind}_{\mathbb{H}_{2}}^{\mathbb{H}} S t \otimes \chi_{0} \simeq \operatorname{Ind}_{\mathbb{H}_{3}}^{\mathbb{H}} S t \otimes \chi_{0}, \\
\operatorname{Ind}_{\mathbb{H}_{2,3}}^{\mathbb{H}} S t \otimes \chi_{0}, & \operatorname{Ind}_{\mathbb{H}_{1,3}}^{\mathbb{H}} S t \otimes \chi_{0}, \\
\operatorname{Ind}_{\mathbb{H}_{1,2}}^{\mathbb{H}} S t \otimes \chi_{0}, & \operatorname{Ind}_{\mathbb{H}_{1,2}}^{\mathbb{H}} \tau_{1} \otimes \chi_{0} .
\end{array}
$$

The isomorphism between the representations induced from $\mathbb{H}_{1}, \mathbb{H}_{2}$, and $\mathbb{H}_{3}$ is a result of the conjugacy of $s_{1}, s_{2}$, and $s_{3}$ in $W$.

These representations are indeed tempered. This can be verified using the following result [BM93, Proof of Theorem 6.4] on the weights of standard modules.

Theorem 7.1 (Barbasch-Moy). If $U$ is a representation of $\mathbb{H}_{P}$, then the weights of $\operatorname{Ind}_{\mathbb{H}_{P}}^{\mathbb{H}} U$ have the form $w \lambda$, where $w \in W^{P}=\left\{w \in W \mid w\left(R_{P}^{+}\right) \subseteq R^{+}\right\}=\{$coset representatives of $W / W_{P}$ of minimal length\}, and $\lambda$ is a weight of $U$.

It is easy to find the minimal length coset representatives of the various $W / W_{P}$ using the Cayley graph and to check that $\left(w \lambda, \omega_{i}\right) \leq 0$ for all such $w$ and all fundamental co-weights $\omega_{i}$.

Since families of tempered representations of affine Hecke algebras are parametrized by representations of the Weyl group, and since there are ten conjugacy classes in $A_{5} \times \mathbb{Z}_{2}$, we expect there to be four other tempered representations which arise as constituents of principal series. There are in fact four additional infinitesimal characters which lead to principal series with full reducibility. Representatives for these four infinitesimal characters are

$$
\begin{aligned}
& \chi_{1}=\sqrt{2}\left(\frac{1}{2}, 4 \gamma-\frac{1}{2}, \frac{1}{2}\right), \\
& \chi_{2}=\sqrt{2}(1,2 \beta, 2 \gamma), \\
& \chi_{3}=\sqrt{2}\left(\beta, \beta+\frac{1}{2}, 2 \gamma\right), \\
& \chi_{4}=\sqrt{2}\left(\beta+1,3 \beta+\frac{1}{2}, 2 \beta\right) .
\end{aligned}
$$

These are the same as the distinguished points in Table 4.13 of [HO] with $k=\frac{1}{2}$, but in the reverse order. At each of these points, the principal series has $8=2^{3}$ factors. and thus at least one tempered constituent by Remark 6.6. Calculations suggested by Rodier's Theorem can be performed at these characters to show that there is in fact exactly one tempered constituent for each and to find the weights and dimensions of these constituents. This is most reasonably done by computer.

It is possible to write procedures which construct the four orbits $W \chi_{i}$ for $i=$ $1,2,3,4$ and take inner products with the co-roots. For those which are $\frac{1}{2}$ another procedure removes the corresponding edge from the list which is used to construct the Cayley graph. After plotting this altered Cayley graph, we can proceed to analyze whether any of the representations corresponding to its connected components are tempered. This is done by choosing the elements of the orbit $W \chi_{i}$ which correspond to the vertex sets for the components, then taking the inner products of these elements with the three co-weights.

The conclusion of these computations is that the four additional tempered representations of $\mathbb{H}\left(H_{3}\right)$ are of dimensions $13,4,3$, and 1, each of which appears as the only tempered constituent of the corresponding principal series $I\left(\chi_{i}\right)$. By 
Remark 6.6, it is easy to find the sequence of signs that parametrize each of these constituents and to check whether the constituent is spherical. The resulting Cayley graphs are shown in Figure 3 and the main results are stated in Theorems 7.2 and 7.3. It remains to be shown that these are all the tempered representations of $\mathbb{H}\left(H_{3}\right)$, as is expected.

Theorem 7.2. There are at least 10 tempered representations of $\mathbb{H}\left(H_{3}\right)$.

Theorem 7.3. The three-dimensional representation, $\rho_{3}$, which is the only tempered constituent of $I\left(\chi_{3}\right)$, is nonspherical.

Proof. By Remark 6.6, it is sufficient to examine the signs in the expression of $\chi_{3}$ as a linear combination of the three appropriate $\check{\beta}_{j}$. Since one of these is negative, we know $\rho_{3}$ is not spherical.

Alternatively, in this case we can argue purely by dimension. The irreducible representations of $W$ have dimensions $1,1,3,3,3,3,4,4,5,5$. Since the action of $\mathbb{C}[W]$ on $I\left(\chi_{3}\right)$ is via the regular representation, the restriction of $\rho_{3}$ to $W$ must be irreducible of dimension 3 and therefore cannot contain the sign representation.

In addition, the computations performed above using Rodier's Theorem make the construction of the representation $\rho_{3}$ straightforward. Begin by taking $\rho_{3}$ to be the twist of the reflection representation tensored with the sign representation on $W$ and write the matrices for $\rho_{3}\left(t_{i}\right)$. The fact that $\rho_{3}$ is scalar on the co-weight $\omega_{1}$ makes it possible to solve the equation $\omega_{1} t_{1}=t_{1}\left(\omega_{1}-\alpha_{1}\right)+\frac{1}{2}$ for $\alpha_{1}$. It is then an
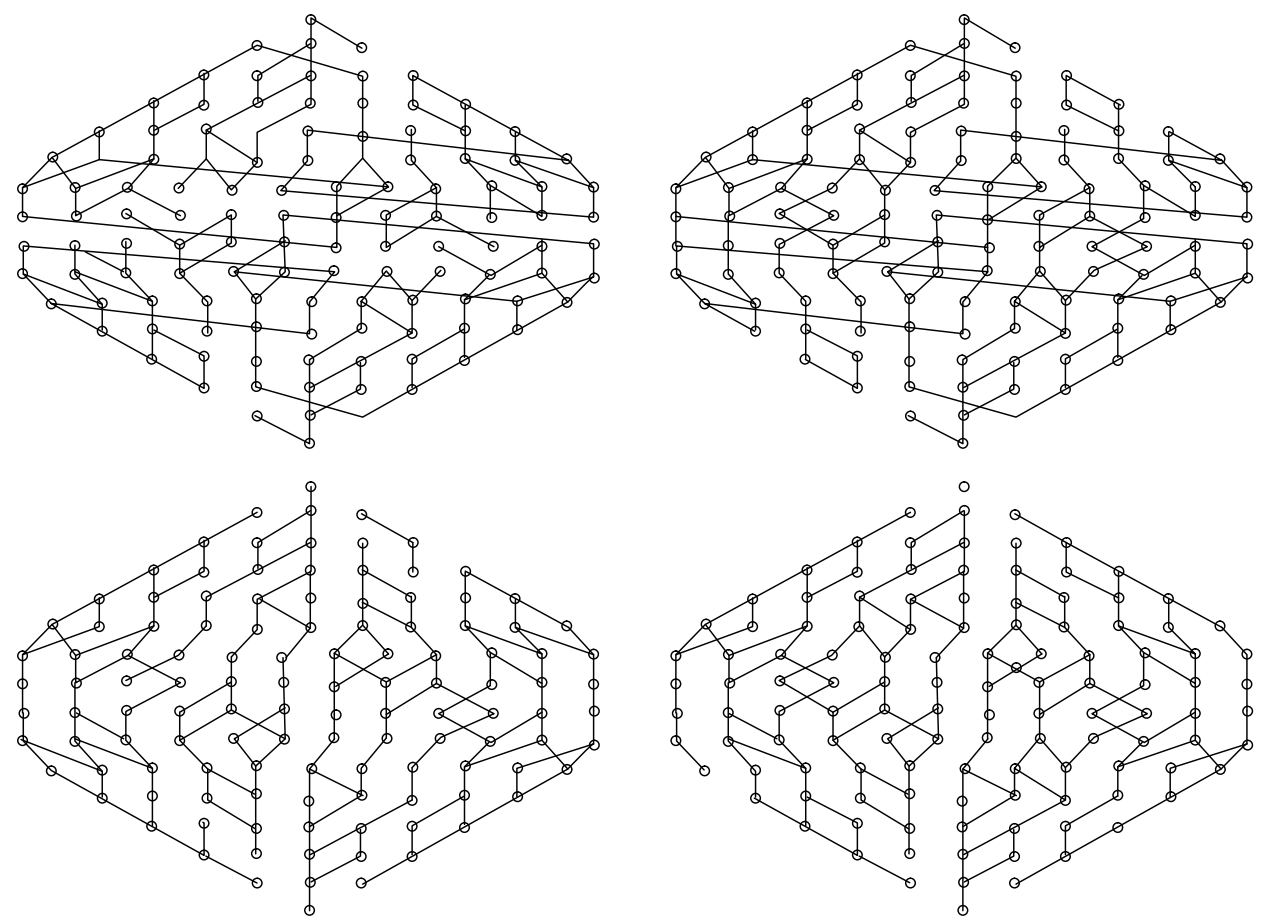

Figure 3. Reduced Cayley graphs $\Gamma\left(\chi_{1}\right), \ldots, \Gamma\left(\chi_{4}\right)$ 
easy matter to solve for $\alpha_{2}$ and $\alpha_{3}$ and verify that the additional relations involved in the definition of $\mathbb{H}\left(H_{3}\right)$ hold as well.

Remark 7.4. For type $H_{4}$, there are four regular distinguished characters listed in Table 4.14 of [HO] (numbers 3,6,10, and 11) at which no spherical cuspidal representations were accounted for. Representatives for these four infinitesimal characters, written with respect to the standard basis of $\mathbb{R}^{4}$ with $\beta$ and $\gamma$ as before and with $\tau=2 \beta$ and $k$ as in Table 4.14 equal to $1 / 2$, are

$$
\begin{aligned}
\chi_{3} & =\sqrt{2}\left(\beta, \gamma+1, \tau-1,3 \tau+\frac{3}{2}\right), \\
\chi_{6} & =\sqrt{2}\left(\frac{1}{2}, 4 \gamma-\frac{1}{2}, \frac{1}{2}, \tau+\frac{5}{2}\right), \\
\chi_{10} & =\sqrt{2}\left(\gamma, \frac{1}{2}, 4 \gamma-1,5 \gamma+\frac{1}{2}\right), \\
\chi_{11} & =\sqrt{2}(4 \gamma-1,-2 \gamma+1,4 \gamma-1, \tau) .
\end{aligned}
$$

We also note here the normalizations chosen for the simple roots and fundamental co-weights.

$$
\begin{array}{ll}
\alpha_{1}=\sqrt{2}\left(\beta, \frac{-1}{2}, \gamma, 0\right), & \omega_{1}=\sqrt{2}(\beta, \beta, \beta, 3 \beta+1), \\
\alpha_{2}=\sqrt{2}\left(-\beta, \frac{1}{2}, \gamma, 0\right), & \omega_{2}=\sqrt{2}\left(\frac{1}{2}, \beta+\frac{1}{2}, \beta, 4 \beta+1\right), \\
\alpha_{3}=\sqrt{2}\left(\frac{1}{2}, \gamma,-\beta, 0\right), & \omega_{3}=\sqrt{2}\left(\frac{1}{2}, \beta, 0,3 \beta+\frac{1}{2}\right), \\
\alpha_{4}=\sqrt{2}\left(\frac{-1}{2},-\beta, 0, \gamma\right), & \omega_{4}=\sqrt{2}\left(0,0,0, \frac{1}{2 \gamma}\right) .
\end{array}
$$

Since the reflection group for $H_{4}$ has 14,400 elements, it was necessary to dramatically improve the efficiency of the procedures described above in order to run computations similar to those above at these four characters. This revealed exactly one tempered constituent among $16=2^{4}$ constituents for each principal series. They have dimensions 4, 20,86 and 284 and are easily verified to be nonspherical using the criterion in Remark 6.6. Further analysis will be required at the 5 remaining nonregular distinguished points for type $H_{4}$ with no spherical cuspidal representations.

These four representations and the representation $\rho_{3}$ in Theorem 7.3 are thus representations which may be expected at the distinguished points referred to in Proposition 4.19 of [HO], but at which there are no spherical cuspidal representations. A second way of interpreting their importance is that they could be viewed as counterexamples to the following extension of a conjecture on Whittaker models from the theory of $p$-adic groups.

The Whittaker model was originally defined for $p$-adic groups in a way which appears to be dependent on the structure of the group. However, a result of Barbasch and Moy [BM94, Theorem 4.4] allows it to be reformulated in a way that can be interpreted purely algebraically on the affine Hecke algebra and thus extended to the general Hecke algebra setting. Using this, we say that a constituent of the principal series $I(\chi)$ has a Whittaker model if and only if it contains the sign representation of $\mathbb{C}[W]$. The analogue of the conjecture then becomes-if $I(\chi)$ has a 
tempered constituent, then some tempered constituent at the infinitesimal character $W \chi$ has a Whittaker model. The fact that each of these representations is the only tempered representation amongst the constituents of their principal series and that each does not contain the sign representation of $\mathbb{C}[W]$ demonstrates that the extension of the conjecture is false for general graded Hecke algebras.

\section{REFERENCES}

[BM93] D. Barbasch and A. Moy, Reduction to real infinitesimal character in affine Hecke algebras, J. Amer. Math. Soc. 6 (1993), no. 3, 611-635. MR 93k:22015

[BM94] D. Barbasch and A. Moy, Whittaker models with an Iwahori fixed vector, Contemp. Math. 177 (1994), 101-105. MR 95j:22024

[Bor76] A. Borel, Admissible representations of a semisimple p-adic group over a local field with vectors fixed under an Iwahori subgroup, Inv. Math. 35 (1976), 233-259. MR 56:3196

[BW80] A. Borel and N. Wallach, Continuous Cohomology, Discrete Subgroups, and Representations of Reductive Groups, Annals of Math. Studies, vol. 94, Princeton University Press, Princeton, 1980. MR 83c:22018

[BZ76] I. Bernstein and A. Zelevinsky, Representations of the groups $G L_{n}(F)$ where $F$ is a nonarchimedean local field, Russian Math. Surveys 31 (1976), 1-68.

[Cas] W. Casselman, Introduction to the theory of admissible representations of $p$-adic reductive groups, unpublished notes.

[Che91] I. Cherednik, A unification of Knizhnik-Zamolodchikov and Dunkl operators via affine Hecke algebras, Invent. Math. 106 (1991), 411-431. MR 93b:17040

[Eve96] S. Evens, The Langlands classification for graded Hecke algebras, Proc. Amer. Math. Soc. 124 (1996), no. 4, 1285-1290. MR 96g:22022

[GB85] L. Grove and C. Benson, Finite Reflection Groups, Springer-Verlag, New York, 1985. MR 85m:20001

[Hel84] S. Helgason, Groups and Geometric Analysis, Academic Press, New York, 1984. MR 86c:22017

[Hil82] H. Hiller, Geometry of Coxeter Groups, Pitman Advanced Publishing Program, Boston, 1982. MR 83h:14045

[HO] G. Heckman and E. Opdam, Yang's system of particles and Hecke algebras, Ann. of Math. 145 (1997), 139-173. CMP 97:07

[Hum90] J. Humphreys, Reflection Groups and Coxeter Groups, Cambridge University Press, Cambridge, 1990. MR 92h:20002

[Jan93a] C. Jantzen, Degenerate principal series for orthogonal groups, J. Reine Angew. Math. 441 (1993), 61-98. MR 94f:22022

[Jan93b] C. Jantzen, Degenerate principal series for symplectic groups, Mem. Amer. Math. Soc. 102, No. 488 (1993). MR 93g:22018

[Kat82] S. Kato, Irreducibility of principal series representations for Hecke algebras of affine type, J. Fac. Sci., Univ. Tokyo, Sect. 1A 28 (1982), no. 3, 929-943. MR 84b:22029

[KL87] D. Kazhdan and G. Lusztig, Proof of the Deligne-Langlands conjecture for Hecke algebras, Invent. Math. 87 (1987), 153-215. MR 88d:11121

[Kri] C. Kriloff, Representations of graded Hecke algebras associated to noncrystallographic root systems, Thesis, University of Michigan (1995).

[Lus88] G. Lusztig, Cuspidal local systems and graded Hecke algebras I, Inst. Hautes Études Sci. Publ. Math. 67 (1988), 145-202. MR 90e:22029

[Lus89] G. Lusztig, Affine Hecke algebras and their graded version, J. Amer. Math. Soc. 2 (1989), no. 3, 599-635. MR 90e:16049

[Lus95] G. Lusztig, Cuspidal local systems and graded Hecke algebras II, Representations of Groups (Providence) (B. Allison and G. Cliff, eds.), vol. 16, Amer. Math. Soc., 1995, pp. 217-275. MR 96m:22038

[Rod81] F. Rodier, Décomposition de la série principale des groupes reductifs p-adiques, Noncommutative Harmonic Analysis and Lie Groups, Lecture Notes in Math. 880 (1981), 408-423. MR 83i:22029 
[Rog85] J. Rogawski, On modules over the Hecke algebra of a p-adic group, Invent. Math. 79 (1985), 443-465. MR 86j:22029

[Ste92] J. Stembridge, A Maple package for root systems and finite Coxeter groups, unpublished documentation, 1992 .

Department of Mathematics, Idaho State University, Pocatello, Idaho 83209-8085

E-mail address: krilcath@isu.edu 